\title{
The Status of the CMS Forward Pixel Detector
}

\author{
Ping Tan* \\ on behalf of the CMS Forward Pixel Collaboration \\ *Fermi National Accelerator Laboratory, P.O. Box 500, Batavia, IL 60510, USA
}

\begin{abstract}
The silicon pixel detector is the innermost component of the CMS tracking system. It provides precise measurements of space points to allow effective pattern recognition in multiple track environments near the LHC interaction point. The end disks of the pixel detector, known as the Forward Pixel detector, are constructed mainly by the US-CMS collaborators. The design techniques, readout electronics, test beam activities, and construction status are reviewed.
\end{abstract}

Keywords: LHC; CMS; Forward Pixel Detector.

PACS: 29.40.Gx.

\section{INTRODUCTION}

The Compact Muon Solenoid (CMS) is one of the detectors being built for the Large Hadron Collider (LHC) [1, 2]. The detector is mainly composed of an all-silicon tracker, an electromagnetic calorimeter, a hadronic calorimeter, and a muon detector. All these components except the muon detector are immersed in a $4 \mathrm{~T}$ superconducting magnetic field. The CMS detector has been designed for multiple-purpose physics studies at hadronic environments. Among those possible studies, the detector is optimized for searching for the Standard Model Higgs boson via $H \rightarrow \gamma \gamma$ decays. Currently, the CMS detector is at the final stage of its construction and is expected to be commissioned in 2007.

The CMS tracking system is an all-silicon detector [3]. It contains both the strip detector and pixel detector. The inner most component is the pixel detector, consisting of three barrel layers and two end disks on each side. The pixel detector provides precise measurements of space points to allow effective pattern recognition in multitrack environments near the LHC interaction point. The end disks, known as the Forward Pixel detector, will be located at a mean distance of $34.5 \mathrm{~cm}$ and $46.5 \mathrm{~cm}$ longitudinally from the interaction point. One of the main challenges in the design of the pixel detector is the expected high level radiation in LHC environment. The pixel detector is required to remain operational with a fluence up to $6 \times 10^{14} \mathrm{n}_{\mathrm{eq}} / \mathrm{cm}^{2}$.

\section{FORWARD PIXEL DETECTOR}

The pixel detector is composed of two-dimensional arrays of pixel cells. The sensor design is required to meet the LHC running environments. The final choice of the 
sensors design is $n+$ in $n$ sensor using the p-stop isolation technique [4, 5]. Multiple guard-rings are used to ensure a high break-down voltage. The dimension of the pixel cell is $100 \mu \mathrm{m} \times 150 \mu \mathrm{m}$. The pixel readout chip (ROC), fabricated in $0.25 \mu \mathrm{m}$ CMOS with radiation-tolerant design, is organized in 26 double columns with 80 rows on each column [6], with a total of 4160 readout channels. There are 18 million readout channels for the Forward Pixel detector. Each readout channel contains a preamplifiershaper, followed by a discriminator. Analog information of the pulse height is read out to improve the position resolution. The expected resolution is $14 \mu \mathrm{m}$ in the tangential direction and $17 \mu \mathrm{m}$ in the radial direction.

The basic detector unit is called a plaquette. Plaquettes are then used to form panels, blades, and then half-disks. Figure 1 shows a flow diagram of the steps in construction. The pixel detector module is formed by connecting a number of ROCs (varies from two to ten) by bump-bonds to a single piece of silicon sensor. A plaquette is then formed by gluing the pixel module on top of a Very High-Density Interconnect (VHDI) flex-circuit which is laminated on a thin layer of silicon substrate. A total of 672 plaquettes are needed to build the Forward Pixel Detector. Due to the geometrical constraints of the panels, five types of plaquettes with different dimensions are needed. Several plaquettes (three or four) are placed on top of a turbine blade shaped HDI circuit to form a panel, with the HDI first laminated to a piece of Beryllium. A total of 192 panels will be built. Panels are mounted to V-shape Aluminum cooling channels, one on each side, to form a blade. The placement of the plaquettes on the panels is positioned so that the blade will have full coverage between the plaquettes (see Figure.1). 12 blades are needed to construct a half-disk. The blades are tilted about $20^{\circ}$ along the tangential direction to increase charge sharing among pixel cells. Half-disks are mounted on carbon-fiber half-cylinders where cooling, power lines and front end electronics are installed. The detector will have two disks on each side. However, the current design allows an upgrade of the detector to a three-disk system, which can extend the coverage in the forward and backward directions.

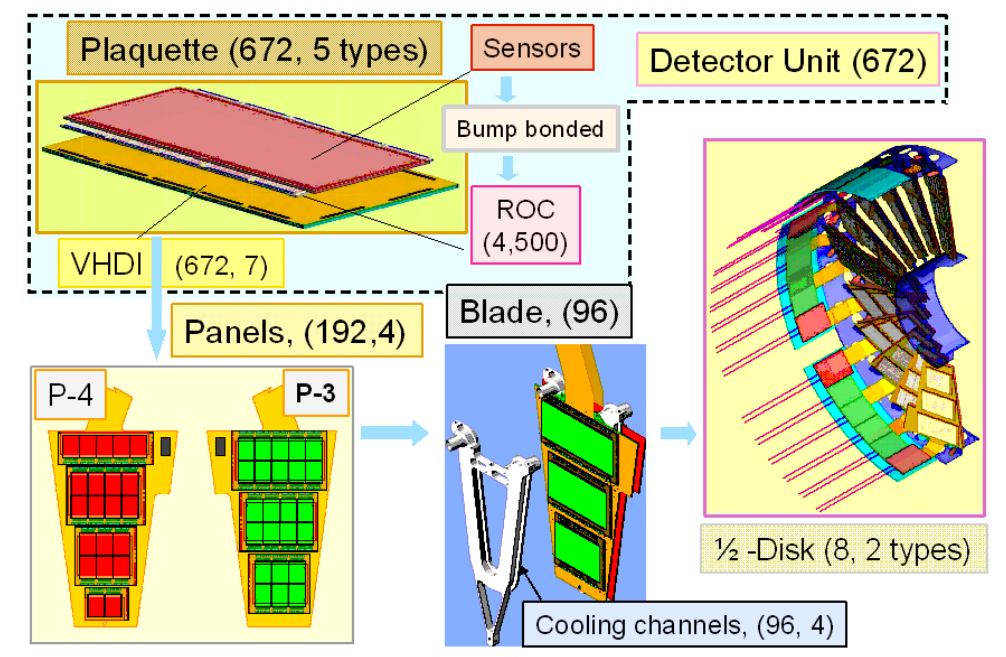

FIGURE 1. The flow diagram of constructing the Forward Pixel detector is shown. The basic detector unit is plaquette. Plaquettes are used to form panels, blades, and half-disks, etc (see text for detailed description). 
CMS has decided to use optical readout for all the sub-detector systems to decrease electrical interference. A front-end controller located in the CMS counting room is used to configure and control the front-end electronics. The control and readout of each panel is realized in the front-end electronics via a token bit manager (TBM). When a trigger comes in, the TBM generates a token and passes it to the ROCs located on the same panel. The corresponding analog data of the triggered event stored in ROCs are then read out. Analog signals are then converted to optical signals in an optohybrid which will then be sent to the counting room where a front-end digitizer is used to decode and reformat the incoming data. The maximum trigger acceptance of the pixel system is $100 \mathrm{kHz}$.

Since the beginning of the project in 1998, many prototypes of the sensor, ROC, and other front-end components have been designed and extensively tested. The performance of the sensor has been tested in real beam environments. In a beam test performed at Fermilab, where preproduction sensors are read out with prototype ROCs, the efficiency of the sensor was measured to be $99.3 \%$ [7]. With $70^{\circ}$ incident tracks, a resolution of $14 \mu \mathrm{m}$ was achieved when charges are shared between two adjacent cells. After the sensors are irradiated with a fluence of $6 \times 10^{14} \mathrm{mip} / \mathrm{cm}^{2}$, the efficiency has been measured in a beam test at CERN to be about $98.3 \%$. These verify that the sensors meet the design requirement. In the ongoing beam tests at Fermilab, the radiation damage to the sensor, ROC, HDI panel and front-end electronics components is being studied in detail. The preproduction sensors and ROCs remain operational at least up to a fluence of $1.3 \times 10^{14} \mathrm{mip} / \mathrm{cm}^{2}$. With the available preproduction parts, recently a panel has been built at Fermilab and we succeeded in reading out this panel. Test stands for sensor/plaquette production tests have been completed and testing plan and schedule are also finalized.

\section{SUMMARY}

The Forward Pixel project has switched from the R\&D phase to production recently. A large number of the production components are being ordered and getting tested for the final assembly. The final production assembly will start in early 2006. The whole detector will be assembled at Fermilab and shipped to CERN in the summer of 2007. The final installation of the Forward Pixel detector is expected in the beginning of 2008, and we expect its performance can bring significant impact on the physics discoveries at LHC era.

\section{REFERENCES}

1. The CMS Collaboration, CMS Tracker Technical Design Report, CERN/LHCC 1998-6, 1998.

2. The CMS Collaboration, Addendum to CMS Tracker Technical Design Report Addendum, CERN/LHCC 2000-16, 2000.

3. F. Hartmann [CMS Silicon Tracker Collaboration], Nucl. Instr. Meth. A 478, 285 (2002).

4. K. Arndt et al., Nucl. Instr. Meth. A 511, 106 (2003).

5. G. Bolla et al., Nucl. Instr. Meth. A 485, 89 (2002).

6. W. Erdmann [CMS Collaboration], Nucl. Instr. Meth. A 549, 153 (2005).

7. G. Bolla et al., Nucl. Instr. Meth. A 501, 160 (2003). 